\title{
Decorrelating Topology with HMC
}

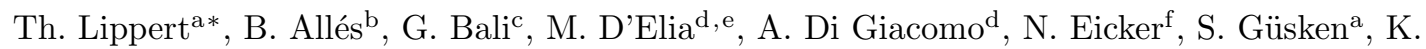 \\ Schilling $^{\text {a,f }}$, A. Spitz ${ }^{\mathrm{f}}$, T. Struckmann ${ }^{\mathrm{a}}$, P. Ueberholz ${ }^{\mathrm{a}}$, and J. Viehoff ${ }^{\mathrm{a}}$ \\ ${ }^{a}$ Department of Physics, University of Wuppertal, D-42097 Wuppertal, Germany \\ ${ }^{\text {b} D i p a r t i m e n t o ~ d i ~ F i s i c a, ~ U n i v e r s i t a ̀ ~ d i ~ M i l a n o ~ a n d ~ I N F N, ~ V i a ~ C e l o r i a ~ 16, ~ I-20133 ~ M i l a n o, ~ I t a l y ~}$ \\ ${ }^{\mathrm{c} I n s t i t u t ~ f u ̈ r ~ P h y s i k, ~ H u m b o l d t ~ U n i v e r s i t a ̈ t, ~ I n v a l i d e n s t r a s s e ~ 110, ~ D-10115 ~ B e r l i n, ~ G e r m a n y ~}$ \\ dDipartimento di Fisica dell'Università and INFN, Piazza Torricelli 2, I-56126-Pisa, Italy \\ ${ }^{\text {e }}$ Department of Natural Sciences, University of Cyprus P.O. Box 537, Nicosia CY-1678, Cyprus \\ ${ }^{\mathrm{f}}$ HLRZ, c/o FZ-Jülich, and DESY-Hamburg, D-52425 Jülich, Germany
}

\begin{abstract}
The investigation of the decorrelation efficiency of the HMC algorithm with respect to vacuum topology is a prerequisite for trustworthy full QCD simulations, in particular for the computation of topology sensitive quantities. We demonstrate that for $\frac{m_{\pi}}{m_{\rho}}$-ratios $\geq 0.69$ sufficient tunneling between the topological sectors can be achieved, for two flavours of dynamical Wilson fermions close to the scaling region $(\beta=5.6)$. Our results are based on time series of length 5000 trajectories.
\end{abstract}

\section{Introduction}

Topology is a fundamental feature of continuum quantum field theories with important bearings on elementary particle physics issues, as there is the proton spin content, the large $\eta^{\prime}$ mass or the role of instantons for the vacuum structure.

Stochastic lattice sampling of QCD configurations must be sufficiently ergodic in the topological sectors of phase space in order to allow for the proper computation of topological quantities. In particular for full QCD, the tunneling rates induced by the hybrid Monte Carlo algorithm between the topological sectors are expected to decrease dramatically when approaching the chiral limit, as known from staggered fermion simulations [1] 3]. For full QCD with dynamical Wilson fermions the actual size of the $\frac{m_{\pi}}{m_{\rho}}$ window accessible by todays simulations is still an open question which we are going to address here [ []

In the last years, various techniques for the reliable computation of topological continuum quantities from the discrete lattice data have been put

\footnotetext{
${ }^{*}$ Talk presented by Th. Lippert
}

forth [5]. In the present investigations, we will just make use of the field theoretical definition [6] of the topological charge densityf

$Q(x)=\frac{g^{2}}{64 \pi^{2}} \epsilon^{\mu \nu \rho \sigma} F_{\mu \nu}^{a}(x) F_{\rho \sigma}^{a}(x)$

with application of cooling [3] as it is not necessary to compute the renormalization of $Q$.

For our investigations, we exploit the SESAM and $T \chi L$ [9] samples consisting of three time histories on lattices of size $16^{3} \times 32$ at $\kappa=0.156$, 0.157 , and 0.1575 , at $\frac{m_{\pi}}{m_{\rho}}$ ratios of $0.839(4)$, $0.755(7)$, and $0.69(1)$, respectively and of configurations on $24^{3} \times 40$ lattices, again at $\beta=5.6$, with $\kappa=0.1575$ and 0.158 , the latter giving rise to $\frac{m_{\pi}}{m_{\rho}} \approx 0.58(2)$. The length of the samples is 5000 trajectories. For $\kappa=0.158$, the length is 3500 trajectories. From these samples, we take sets of $N=200$ 'decorrelated' configurations. ${ }^{2}$ Alternatively, we follow Smit and Vink $[7$ avoiding cool-
ing. With $G$ being the gyark propagator we compute $Q$
via stochastic estimates 8 :
$Q=m \kappa_{P}\left\langle\operatorname{Tr}\left(\gamma_{5} G\right)\right\rangle_{U}$. 
Table 1

First part: mobility $D_{25}$. Second part: mobility normalized by dividing through square root of the volume.

\begin{tabular}{l||c|c|c|c|c|c}
$\kappa$ & .156 & .157 & .1575 & 0.1580 & quenched \\
\hline \multicolumn{7}{c}{$D_{25}$} \\
\hline $16^{3} \times 32$ & 2.8 & 2.5 & 1.9 & - & - \\
\hline $24^{3} \times 40$ & - & - & 3.8 & 2.8 & - \\
\hline $16^{4}$ & - & - & - & - & 1.2 \\
\hline \multicolumn{7}{c}{$D_{25} / \sqrt{V}$} \\
\hline $16^{3} \times 32$ & 2.8 & 2.5 & 1.9 & - & - \\
\hline $24^{3} \times 40$ & - & - & 1.9 & 1.4 & - \\
\hline $16^{4}$ & - & - & - & - & 1.7
\end{tabular}

\section{Results}

In Fig. (四) we present the time series of the topological charge $Q$ from the five samples. The first three canvases are computed from SESAM ensembles measured on lattices of size $16^{3} \times 32$, the next two are from $T \chi \mathrm{L}$ ensembles from $24^{3} \times$ 40 systems. We analyzed every 25 th configuration. In the first three graphs, the results from the gluonic and fermionic measurements are superimposed demonstrating nice agreement. For all three SESAM masses, the figure tells us that HMC can create sufficient tunneling in the SESAM $\frac{m_{\pi}}{m_{\rho}}$ window.

We quantify the tunneling between the topological sectors defining the mobility for tunneling as

$$
D_{d}=\frac{1}{N} \sum_{i=1}^{N}|(\tilde{Q}((i+1) \cdot d)-\tilde{Q}(i \cdot d))| .
$$

Tab. (1i) demonstrates that the mobility is decreasing when going more chiral.

With the $24^{3} \times 40$ system, at $\kappa=0.1575$, we can assess the volume dependence. Note that $Q$ is an extensive quantity. Still we find the tunneling rates sufficient, however, with smaller quark mass, at $\kappa=0.158$, the mobility decreases. As we expect the mobility to scale approximately with the square root of the volume, we normalize the numbers accordingly, see the second part of Tab. (11).

Analyzing the time history on every second trajectory (at $\kappa=0.1575$ ) we find very frequent tun-

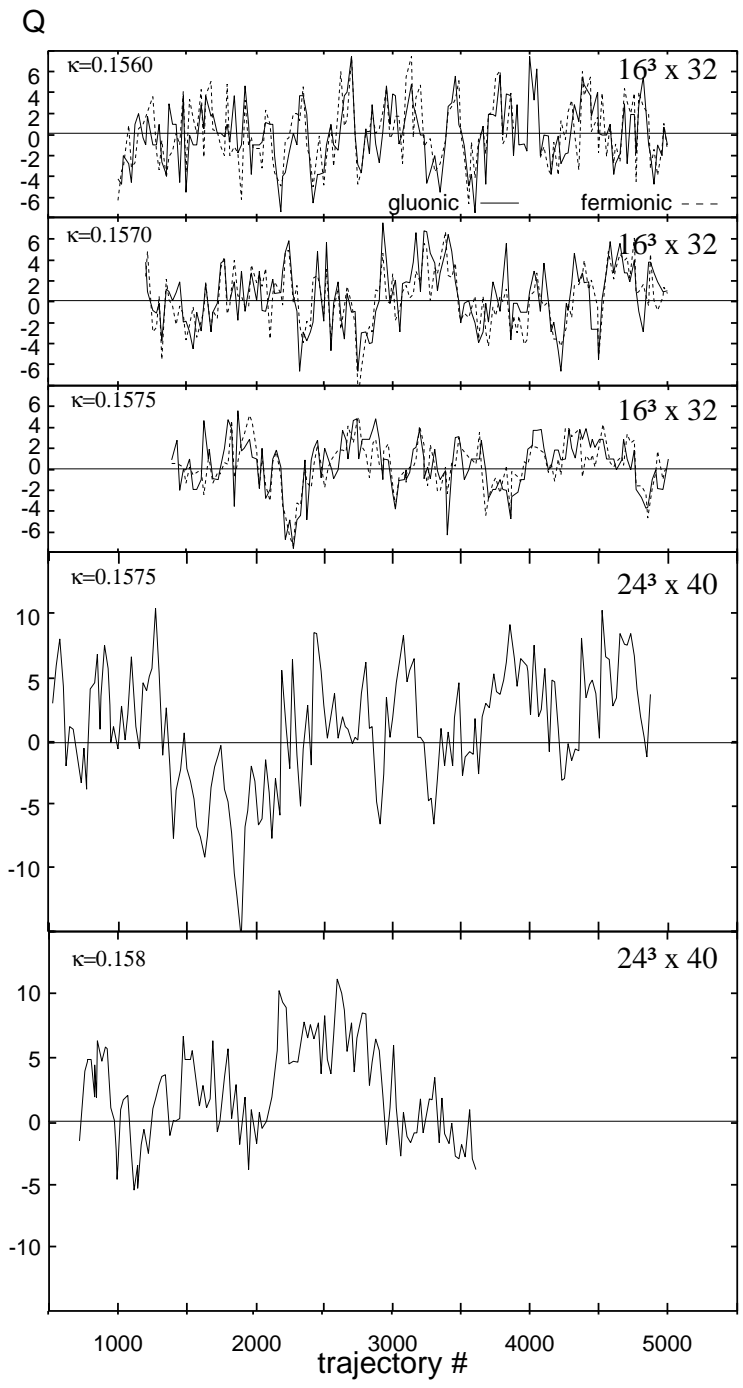

Figure 1. Time series of unrenormalized topological charge. We plot series from 3 SESAM and 2 $\mathrm{T} \chi \mathrm{L}$ ensembles.

neling, see Fig. (2). On this series, we can compute the autocorrelation function. The exponential autocorrelation time is $\tau_{\text {exp }}=80(10)$. The integrated autocorrelation time turns out to be $\tau_{i n t}=54(4)$, compatible with the decorrelation of other observables as found in [10]. 


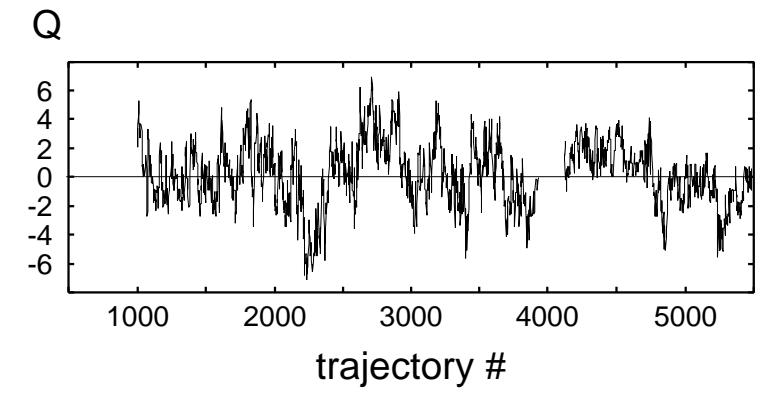

Figure 2. Fine scan of unrenormalized $Q$ for $\kappa=$ 0.1575 on the $16^{3} \times 32$ lattice.

Histogramming Fig. (2) we find a smooth Gaussian, see Fig. (3). This is of course less pronounced for the small decorrelated samples of size 200, see Fig. (3), however, the distributions are still well peaked at $Q=0$.

\section{Summary}

As the main result, we find that HMC tunnels well between the vacuum sectors for $\frac{m_{\pi}}{m_{\rho}} \geq 0.69$. Autocorrelation times are slightly larger than for the yet worst case observable, the minimal eigenvalue of the Wilson fermion matrix. Therefore, we are confident to be able to carry out reliable computations of hadronic properties related to topology, like the proton spin content and the topological susceptibility for $\frac{m_{\pi}}{m_{\rho}}$-values down to 0.69 .

\section{REFERENCES}

1. Y. Kuramashi et al., Phys. Lett. B313 (1993) 425.

2. M. Mueller-Preussker, in Proc. of XXVI Int. Conf. on High Energy Physics, Dallas 1992, Ed. J.R. Sanford, AIP Conference Proceedings No. 272, 1545 (1993)

3. B. Allés, G. Boyd, M. D'Elia, A. Di Giacomo, and E.Vicari, Phys. Lett. B389 (1996) 107.

4. see B. Allés et al.: hep-lat/9803008, to appear in Phys. Rev. D.

5. B. Allés, M. D'Elia, A. Di Giacomo, and R.

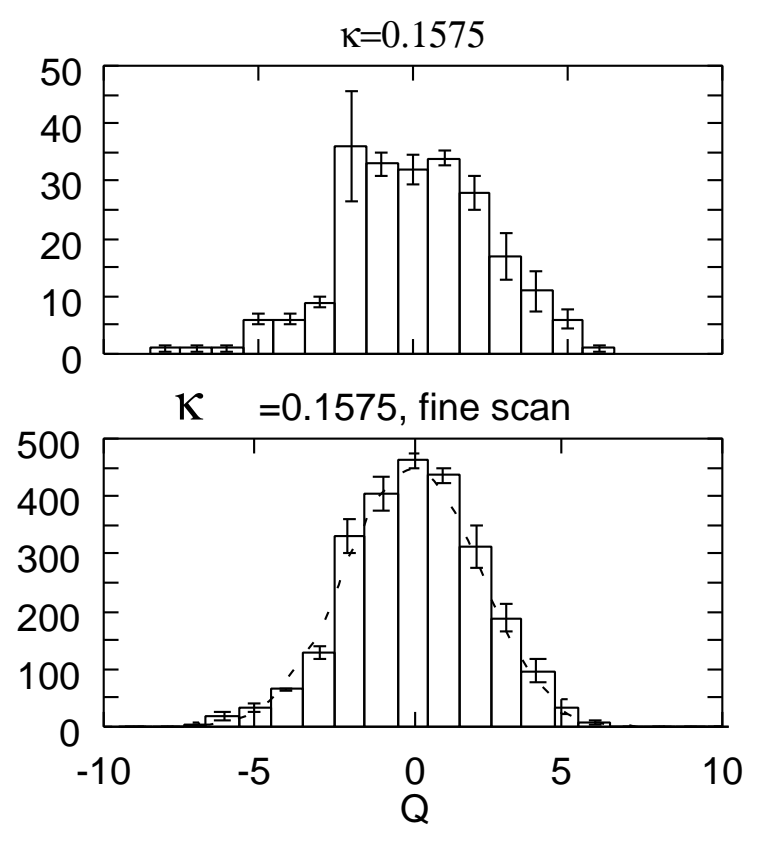

Figure 3. Histograms of the unrenormalized topological charge computed from Eq. (1)

Kirchner, A Critical Comparison of Different Definitions of Topological Charge on the Lattice, hep-lat/9711026.

6. M. Campostrini, A. Di Giacomo, and H. Panagopoulos, Phys. Lett. B212 (1988) 206; B. Allés, M. Campostrini, A. Di Giacomo, Y. Gündüc, and E. Vicari, Phys. Rev. D 48 (1993) 2284.

7. J. Smit and J. C. Vink, Nucl. Phys. B286 (1987) 485.

8. J. Viehoff et al.: these proceedings.

9. Th. Lippert, G. Bali, N. Eicker, L. Giusti, U. Glässner S. Güsken, H. Hoeber, P. Lacock, G. Martinelli, F. Rapuano, G. Ritzenhöfer, K. Schilling, G. Siegert, A. Spitz P. Ueberholz, and J. Viehoff, Nucl. Phys. B Proc. Suppl. 60A (1998) 311.

10. Th. Lippert et al., Nucl. Phys. B Proc. Suppl. 63 (1998) 946. 